\title{
Radioactive Isotope Evaluation of Coronal Leakage after Endodontic Treatment in Teeth Restored with Three Different Intracoronal Restorative Materials: An invitroStudy
}

\author{
${ }^{1}$ Keshava Prasad BS, ${ }^{2}$ Sunu Sudhakaran \\ ${ }^{1}$ Professor, Department of Conservative Dentistry and Endodontics, DA PM RV Dental College, Bengaluru, Karnataka, India \\ ${ }^{2}$ Senior Lecturer, Department of Conservative Dentistry and Endodontics, NSVK SV Dental College, Bengaluru, Karnataka, India
}

Correspondence: Keshava Prasad, Professor, Department of Conservative Dentistry and Endodontics, DA PM RV Dental College CA 37, 24th Main, JP Nagar, 1st Phase, Bengaluru-560078, Karnataka, India, e-mail : keshavprasad72@gmail.com

\section{ABSTRACT}

The aim of this study was to compare and evaluate the coronal microleakage following endodontic treatment of teeth restored with three different intracoronal restorations using calcium- 45 radioisotope and autoradiography. A total of 48 extracted human mandibular premolars with single canals were divided into three experimental groups of 16 specimens each. The teeth were sectioned horizontally at the cementoenamel junction and at the middle-third of the root, orifices and canals were enlarged with Gates Glidden bur and finished using $\mathrm{K}$ files, and obturated with gutta-percha cones using lateral condensation technique. $3 \mathrm{~mm}$ of gutta-percha were removed from the coronal part of the teeth to prepare a clean $3 \mathrm{~mm}$ deep cavity for the coronal restorative material. Group 1 consisted of teeth restored with Ketac Molar Easymix; group 2 with Miracle Mix and group 3 with Filtek Z350. The teeth were then thermocycled and tested for microleakage using calcium-45 radioisotope. The results were analyzed using Kruskal-Wallis test and Mann-Whitney test. Filtek Z350 exhibited the least leakage followed by Ketac Molar Easymix and Miracle Mix, and the microleakage among the three restorative materials was statistically significant.

Keywords: Autoradiography, Coronal microleakage, Radioisotope, Restorative materials.

\section{INTRODUCTION}

Endodontic treatment is essentially performed to prevent and control infections of the pulpal and periapical tissues. The outcome of the endodontic therapy depends on the method and quality of instrumentation, irrigation, disinfection, threedimensional obturation of the root canal and a good intracoronal restoration. The concept of coronal leakage having an effect on the outcome of root canal therapy has been known for nearly a century. Early endodontic research focused on the qual ity of the preparation and obturation to ensure long-term treatment success, and the effects of poor coronal restorations on endodontic outcomes received little attention. ${ }^{1}$ On completion of root canal obturation, it is necessary to have an adequate coronal seal by placement of a suitable restorative material which seals the access cavity. Hence, the use and quality of restorative materials for coronal restoration determines the success or failure of the root canal treatment. These materials serve to seal the tooth preventing the entry of fluids, microorganisms and other debris into the root canal space. ${ }^{2}$ Bacteria have been shown to play an important role in endodontic failures. It has also been shown that after normal root canal cleansing and filling, the number of living residual microorganisms is effectively diminished and barring a faulty seal or continuing source of microorganisms, the body will eliminate and repair any pre-existing infection. ${ }^{3}$

Microleakage as related to endodontics refers to the movement of fluid and microorganisms along the interface of dentinal walls and the root filling material or through voids within the root filling material. $M$ any techniques have been devised to test the cavity sealing properties of restorations; like the use of dyes, chemical tracers, radioactive isotopes, air pressure, bacteria, neutron activation analysis, scanning electron microscopy, artificial caries technique and electrical conductivity. ${ }^{4}$ One widely used method of evaluating microleakage is the use of radioisotope and determining the depth of penetration by autoradiography. The distance of radiopacity is measured as an indicator of how deep the isotope leaked between the tooth walls and the restoration. Deeper radiopacity is an area where microleakage is considered pronounced. The isotopes used are ${ }^{45} \mathrm{Ca},{ }^{55} \mathrm{M} \mathrm{n},{ }^{131} \mathrm{I},{ }^{22} \mathrm{Na},{ }^{35} \mathrm{~S}$ and Dysprosium. Calcium is most popular, since it is readily available, has affinity towards calcium of tooth structure, and is a low beta emitter and permits detection of minute amount of leakage due to their smaller particle size.

The purpose of this study was to assess the coronal microleakage of three different intracoronal restorative materials- K etac M olar Easymix (3M ESPE), M iracle M ix (GC corporation) and Filtek Z350 (3M ESPE) using radioactive isotope cal cium- 45 .

\section{MATERIALS AND METHODS}

A total of 48 human single rooted mandibular premolar teeth were scaled and immersed in $5.25 \%$ sodium hypochlorite for 15 minutes to remove tissue remnants. The teeth were then sectioned horizontal ly at the cementoenamel junction and at the middle-third of the root with a tapered fissure carbide bur. Pulp 
tissue was removed from the root canals with barbed broaches. The orifices and canals were enlarged with a size 3 Gates Glidden bur (ISO 90) and finished using K files. Pulp chamber and canals were irrigated with $5.25 \%$ sodium hypochlorite and rinsed with sterile water for 1 minute. Canals were then dried with paper points and obturated with gutta-percha cones using lateral condensation technique. Gutta-percha was cut at the canal orifice with a heated instrument and condensed vertically right at the orifice. The teeth were then divided into three groups of 16 teeth each depending on the coronal restorative material to be used.

$3 \mathrm{~mm}$ of gutta-percha was removed from the coronal part of the teeth using a marked heated plugger to prepare a clean $3 \mathrm{~mm}$ deep cavity. Group 1- the cavity was treated with 10\% polyacrylic conditioner for 10 seconds to remove the smear layer, rinsed and mildly air dried and restored with K etac M olar Easymix according to the manufacturer's instructions. G roup 2 was similarly preconditioned and restored with Miracle according to manufacturer's instructions. Group 3- here an additional $1 \mathrm{~mm}$ of gutta-percha was removed from the canal to make room for a layer of glass ionomer cement to serve as a barrier between the obturation and the nanocomposite material. The cavity was etched with $37 \%$ phosphoric acid for 10 seconds, rinsed for 20 seconds and then bonding agent Prime and $B$ ond NT was applied and cured for 20 seconds. The Filtek Z350 was placed into the prepared cavity in small increments and light-cured for 20 seconds according to the manufacturer's instructions.

All specimens were stored at room temperature in distilled water for 24 hours, and then subjected to thermocycling at $4 \pm 2,55 \pm 2$, and $37 \pm 2^{\circ} \mathrm{C}$ for 100 cycles. The dwell time in each bath and the time interval at room temperature between baths was 20 seconds. The teeth in all specimens received three layers of nail polish leaving only the area of the canal orifice exposed.

The coated teeth were immersed for 24 hours in Ca-45 solution, which was diluted with distilled water to give a solution of $0.1 \mathrm{mCi} / \mathrm{ml}$. A fter removal from the solution, the teeth were rinsed for one hour in running water and sectioned mesiodistally using diamond disc for autoradiographic examination. The two sections obtained per tooth were placed on K odak film and left for 24 hours exposure in the dark. The overall microleakage pattern of each tooth was then examined and a leakage score was assigned and demonstrated schematically.

The microleakage scores were:

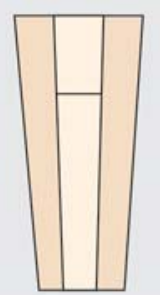

0

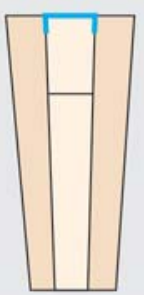

1

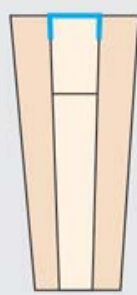

2

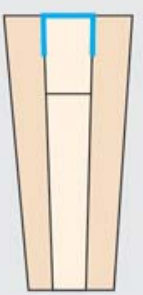

3
$0=$ No evidence of isotope at the interface of the canal wall and the restorative material
$1=$ Slight evidence of isotope penetrating through the canal wall $(<1 \mathrm{~mm})$

$2=$ M oderate evidence of isotope penetrating the canal wall $(1-2 \mathrm{~mm})$ $3=$ Gross evidence of isotope penetrating the canal wall $(>2 \mathrm{~mm})$.

\section{RESULTS}

The autoradiography of samples restored with Filtek Z350 demonstrated 11 samples with score 0 and five samples with score $1 ; \mathrm{K}$ etac $\mathrm{M}$ olar Easymix demonstrated five samples with score 0 , eight samples with score 1 and three samples with score 2; and M iracle M ix group demonstrated seven samples with score 2 and nine samples with score 3 (Figs $1 A$ to C).

Statistical analysis using $\mathrm{K}$ ruskal-W allisand $\mathrm{M}$ ann-W hitney test revealed that there is a significant difference between the different materials with respect to microleakage scores $(p<0.001)$.
Score 0

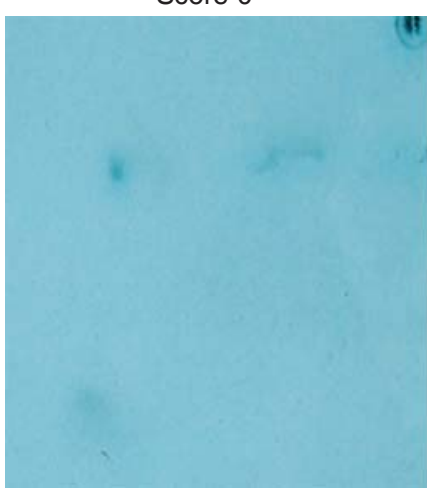

Fig. 1A: Autoradiograph of Filtek Z350 specimen

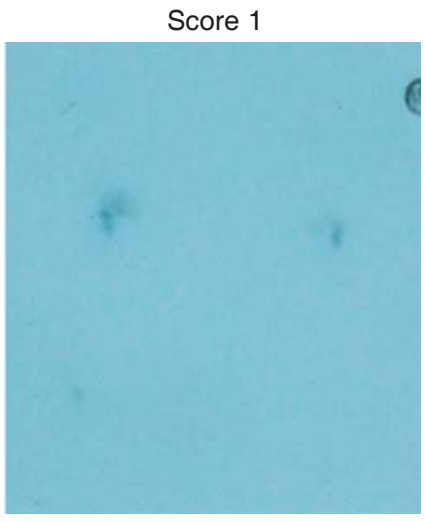

Score 2

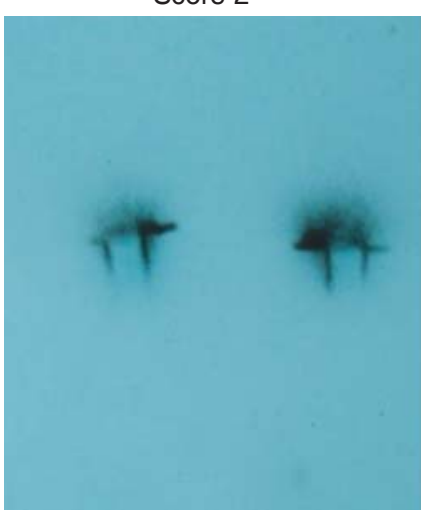

Score 3

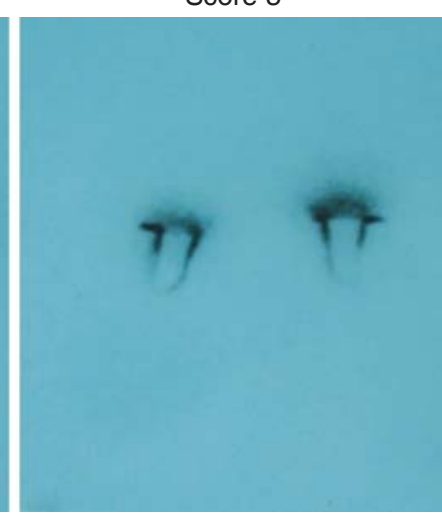

Fig. 1B: Autoradiograph of Miracle Mix specimen

Score 0

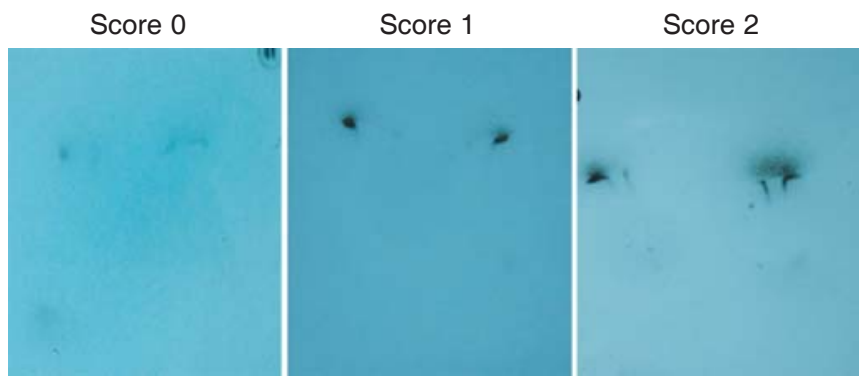

Score 2

Fig. 1C: Autoradiograph of Ketac Molar Easymix specimen 
Radioactive Isotope Evaluation of coronal Leakage after Endodontic Treatment in Teeth Restored

\section{DISCUSSION}

Endodontic treatment is aimed at healing or preventing apical periodontitis. A $n$ infected root canal has to be cleaned, shaped and filled with a three-dimensional seal. This filling together with the intracoronal restoration should prevent oral bacteria from penetrating the apical tissue. ${ }^{1} \mathrm{~F}$ inal restorative materials should protect the root canal system if placed properly. If a seal is not maintained, the success of root canal therapy may be jeopardized when obturated root canals are exposed to the oral microbiota. If microbial cells and their products reach the periradicular tissues, they can induce and/or perpetuate periradicular disease. ${ }^{5}$ In a study using obturated canals exposed to artificial saliva followed by Pelikan ink, found leakage in as little as 3 days. A nother study showed that root canals obturated with gutta-percha and Roth sealer, either by lateral or vertical condensation, demonstrated apical leakage within 30 days of coronal exposure to saliva. B acteria and their byproducts have also been shown to penetrate root canal obturating materials and influence periapical tissues and outcomes for success. The evaluation of bacterial endotoxin from A ctinobacillus to pass through obturated root canals revealed that it was able to pass through the root canals within 20 days. ${ }^{6}$

In this study, cal cium-45 in the form of cal cium chloride at a concentration $0.1 \mathrm{mCi} / \mathrm{ml}$ was used to assess the microleakage. The use of isotopes permits detection of minute amount of leakage, as the smaller isotope molecules measure only $40 \mathrm{~nm}$ compared to the smaller dye particles $(120 \mathrm{~nm})$. A Iso radioactive isotopes have the advantage over dyes as tracers, for their presence can be readily detected in very small concentrations. ${ }^{4}$

The autoradiography of samples restored with Filtek Z350 resulted in 11 samples with no penetration, five samples with slight evidence of penetration; K etac M olar Easymix showed five samples with no penetration, eight samples with slight penetration and three samples with moderate penetration; $M$ iracle M ix showed seven samples with moderate penetration and nine samples with gross penetration. On statistical analysis, it was found that Miracle Mix exhibited the most leakage followed by K etac M olar Easymix and Filtek Z350.

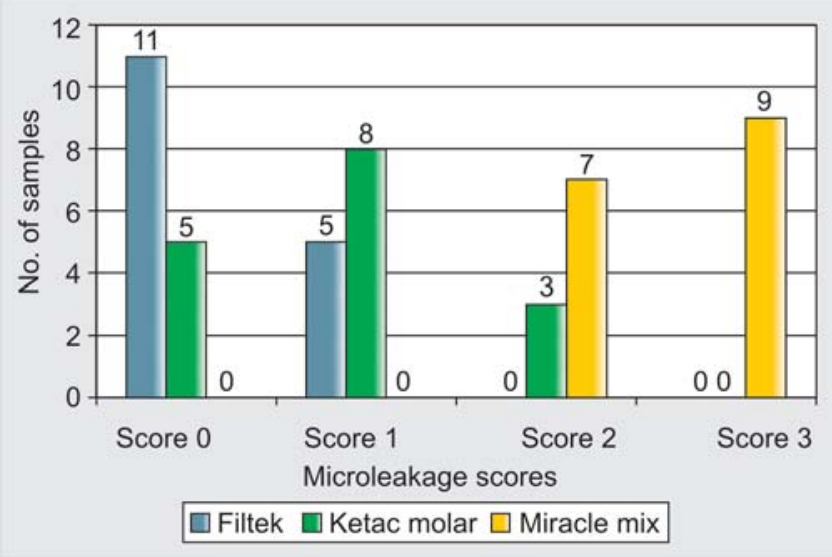

Graph 1: The comparative distribution of microleakage scores in all three groups
The samples of $\mathrm{K}$ etac M olar Easymix was conditioned with $10 \%$ polyacrylic acid before restoration. This was done to remove the smear layer and hence attain an improved seal of the glass ionomer cement to the dentin. However, in spite of using the conditioner, K etac M olar E asymix exhibited leakage in 11 samples, the probable reason being shrinkage of the material upon setting, resulting in a potential avenue for microleakage. These findings could be corroborated to those obtained in a previous study, which revealed a gap observed between glass ionomer and dentinal wall and related it to the shrinkage of the material on setting. ${ }^{7}$

M iracle M ix showed the most leakage, which could al so be explained due to shrinkage of the glass ionomer component, and also due to the fact that silver alloy does not chemically bond to the tooth structure and due to the inherent difference in coefficient of thermal expansion between the tooth and silver alloy ( 8.3 for dentin versus 25 for silver alloy).

The results of this study with Filtek Z350 performing the best, may be explained by the volumetric content of inorganic particles, as the filler content and size directly determine the physical and mechanical properties of composite resin. $\mathrm{Nano}$ composite Filtek Z350 has $78.5 \%$ weight or $59.5 \%$ volume of filler content according to the manufacturer. Due to the reduced dimension of the particles and to a wide size distribution, an increased filler load can be achieved with a consequence of reducing the polymerization shrinkage and thus increasing the marginal adaptation thereby reducing the microleakage. A Iso, the use of latest generation bonding agent Prime and B ond NT, based on nanotechnology, has shown the ability to penetrate dentinal tubules through hydrophilic wetting. By using such a primer the resin is drawn into the tubules creating an intimate hybrid layer and resin tags. This penetration of resin into the dentin creates a mechanical interlocking that would have resisted the penetration of radioisotope thereby giving the best protection against coronal microleakage.

Within the limits of this study, Filtek Z350 gave the best results, thus proving itself to be an ideal material to restore the tooth after endodontic treatment to achieve an adequate seal from coronal microleakage.

\section{ACKNOWLEDGMENTS}

The authors wish to express their sincere gratitude to Dr Saroja, Head of Department, Nuclear M edicine, Victoria Hospital, Bengaluru, and Mr Tejaswi, Statistician for their technical support.

\section{REFERENCES}

1. Claudia R B arthel, Stefan Zimmer, Ralf W ussogk, J ean-Francois Roulet. L ong-term bacterial leakage along obturated roots restored with temporary and adhesive fillings. J Endod 2001; 27(9):559-62.

2. EV Cruz, Y Shigetani, K Ishikawa, K K ota, M I waku. A Iaboratory study of coronal microleakage using four temporary restorative materials. Int Endod J 2002;35:315-20. 
3. Nasrin Roghanizad, J efferson J J ones. Evaluation of coronal microleakage after endodontic treatment. J Endod 1996;22(9):471-73.

4. Alani $A H$, Toh CG. Detection of microleakage around dental restorations: A review. O per Dent 1997;22:173-85.

5. JF Siqueira J r. A etiology of root canal treatment failure: Why well-treated teeth fail? Int Endod J 2001;34:1-10.
6. Gary A W olanek, R obert J Loushine, N orman R W eller, Frank Kimbrough, K eith $\mathrm{R}$ V olkmann. In vitro bacterial penetration of endodontically treated teeth coronally sealed with a dentin bonding agent. J Endod 2001;27(5):354-57.

7. Klara S Alperstein, Heber T Graver, Richard CB Herold $M$ arginal leakage of glass ionomer cements restorations. J Prosthet Dent 1983;50(6):803-07. 